\title{
Semiconductor Laser in Distributed Optical Feedback Regime
}

\author{
Faisal Ahmed Memon ${ }^{1,2} \cdot$ Francesco Morichetti $^{1} \cdot$ Zulfiqar Ali Arain $^{2,3}$ • \\ Umair Ahmed Korai ${ }^{2,4}$ - Andrea Melloni ${ }^{1}$
}

(C) Springer Science+Business Media, LLC, part of Springer Nature 2018

\begin{abstract}
In this work, we first analyse the behaviour of semiconductor laser in the presence of weakto-moderate feedback from a single (lumped) and double external cavity setting. Analysis of laser diode in dynamic double cavity configuration shows that the dynamic double cavity does not disturb the stability of the laser device, but it introduces a considerable shift in the emission frequency that is directly related to the feedback strength and the relative phase difference between the phase of the two mirrors, and the phase of electric field inside the laser diode. Conditions for maximum and minimum impact of the laser emission wavelength have been derived. Addressing the issue of distributed optical feedback, optical feedback produced by more than two external mirrors has been also studied. To this aim we have first generalized the Lang and Kobayashi deterministic rate equations model to arbitrary number of external reflectors and provide the corresponding steady-state solution, which is done for the first time. To the best of our knowledge, this kind of study is carried out for the first time.
\end{abstract}

Keywords Semiconductor laser · Distributed optical feedback · Lang-Kobayashi model · Optical communications

Electronic supplementary material The online version of this article (https://doi.org/10.1007/s11277-0185931-y) contains supplementary material, which is available to authorized users.

Faisal Ahmed Memon

faisalahmed.memon@polimi.it; faisal.memon@faculty.muet.edu.pk

1 Dipartimento di Elettronica, Informazione e Bioingegneria (DEIB), Politecnico di Milano, Via Ponzio 34/5, 20133 Milan, Italy

2 Department of Telecommunications Engineering, Mehran University of Engineering and Technology, Jamshoro 76062, Pakistan

3 Beijing University of Posts and Telecommunication, 10 Xitucheng Rd, Haidian, Beijing, China

4 Department of Electronic and Electrical Engineering, University of Strathclyde, Glasgow G1 1XW, UK 


\section{Introduction}

The dynamics of semiconductor laser subject to optical feedback are explored since a long time. Over past two decades, researchers have produced remarkable results which assist in understanding the behaviour of other nonlinear systems, such as, fluid dynamics, biology, and chemistry, which also exhibit similar phenomena. Nevertheless, laser diode as a source is widely used in optical communication systems, spectroscopy, and optical measurement systems. Therefore, understanding the behaviour of such device with optical feedback is of prime importance. So far, several different types of feedback regimes are discovered which strongly depends on feedback strength and length of external cavity, in addition to other laser diode's parameters. Beginning with Lang-Kobayashi model [1], which showed that feedback induces irregular peaks in output intensity and shifts in emission frequency of a semiconductor laser. Then, Tkach and Chraplyvy [2] described five distinct regimes occurring in a laser device based on the length of external cavity and feedback magnitude. The five regimes reveal linewidth narrowing and broadening in the first, mode hopping in the second, locking of a laser on external cavity induced frequency in the third, coherence collapse (CC) in the fourth, and stable operation with narrow linewidth in the fifth regime. Following, Sano [3] demonstrated low frequency fluctuations (LFF) phenomenon in a laser diode due to optical feedback when the external cavity is long and thus, is considered as long cavity regime (LCR). Recently, Heil et al. [4] reported another type of regime which they call the short cavity regime. According to their work, a laser device with short external cavity length exhibits regular pulse packages (RPP) which form low-frequency state with underlying fast, regular intensity pulsations. Along with examining the effects of optical feedback on a laser device, intense research work has been carried out to control the deleterious effects induced by feedback, such as to stabilize the laser diode, and to make positive use of feedback in sophisticate applications such as chaotic communications [5]. The recent works considers only a single-concentrated external reflector which provides optical feedback [6-8].

In this work, we reproduce the first four regimes of [2] as we work on weak-to-moderate optical feedback and consider LK scheme [1, 9, 10], hence the fifth regime is beyond the scope of our research work. Then the LK model is extended to include an arbitrary number of mirrors and examine the effects of phase space and feedback strength of short-to-long external cavities. Finally, distributed reflectors are inserted in the LK model to observe the non-linear dynamics of laser system induced by distributed feedback regime and a comprehensive report of results along with parametric table is presented.

\section{Theoretical Analysis}

The configuration of a laser diode of the cavity length 1 with an external lumped reflector is shown in Fig. 1a. $R_{1}$ and $R_{2}$ are power reflectivities of the device facets, and $R_{3}$ is power reflectivity of external mirror placed at a distance L. A single-mode semiconductor laser subject to weak-to-moderate level of feedback can be represented in terms of LangKobayashi model [1]. In the model, evolution of the electric field in the laser device is described and the influence of feedback is incorporated by addition of a time-delayed field term with coupling strength $k$. Assuming the complex E-field of the form of an envelope $\mathbf{E}(\mathrm{t})$ modulated by fast oscillating optical field term $\mathrm{e}^{(\mathrm{iwot})}$, where $\mathrm{w}_{\mathrm{o}}$ is the center frequency, 

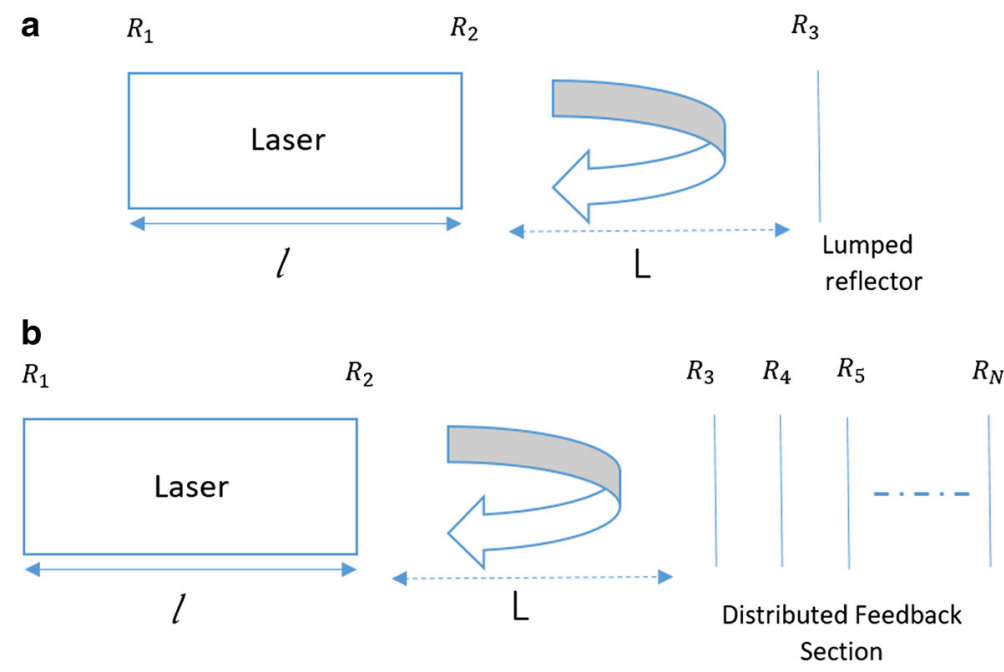

Fig. 1 a Laser cavity with feedback from a lumped reflector and $\mathbf{b}$ laser in distributed feedback regime with $\mathrm{N}$ external reflectors

$$
\begin{gathered}
\frac{d}{d t} E(t)=\frac{1}{2}(1+i \alpha)\left[G_{n}(N, P)-\Gamma_{o}\right] E(t)+\tilde{k} E(t-\tau) e^{-i w_{o} \tau} \\
\frac{d}{d t} N(t)=-\gamma N(t)-G(N, P) P(t)+p \frac{J_{t h}}{e} \\
G(N, P)=\frac{\xi\left(N-N_{t}\right)}{1+\varepsilon P}, P(t)=|E(t)|^{2}
\end{gathered}
$$

The rate Eqs. (1)-(3) are known as L-K equations [1] and have been taken from [12]. The Eqs. (1)-(3) constitute our numerical model which is used throughout this research work to study the dynamics of semiconductor laser with optical feedback and produce the appropriate results. However, these equations take into account a single reflector and the extension of this model for two or more (distributed) reflectors that is the focus of this paper will be presented in the following sub-section. The description of the parameters used in these equations is as follows; $\mathrm{E}(\mathrm{t})$ is a slowly varying electric-field inside the laser diode cavity, $\mathrm{N}(\mathrm{t})$ is the number of carrier, $G_{n}(N, P)$ is the optical gain accounts for amplification by stimulated emission mechanism, $\tilde{k}$ is the feedback rate, $\tau$ is the delay time in the external cavity, $\mathrm{w}_{\mathrm{o}}$ is the angular frequency of the isolated-laser diode, $\alpha$ is the linewidth enhancement factor, $\mathrm{p}$ is the pump current in the units of isolated-laser threshold $\mathrm{J}_{\mathrm{th}}$, and $\mathrm{e}$ is the electronic charge. The effect of optical saturation of the active medium in the laser is included by use of the nonlinear gain parameter $\varepsilon$, and $\xi$ is the differential gain. $1 / \gamma$ and $1 / \Gamma_{o}$ are the carrier and photon lifetimes and $N_{t}$ is the carrier number at transparency, respectively. The feedback term $\tilde{k}$ is the significant addition made by Lang and Kobayashi [1] that includes the effects of the time delayed optical feedback. The coefficient of coupling $\tilde{k}$ is: 


$$
\tilde{k}=\frac{c\left(1-R_{2}\right) \sqrt{R_{3} / R_{2}}}{2 \eta l}=\frac{k}{\tau_{\text {in }}}
$$

The steady state solutions of LK model can be derived from the rate Eqs. (1)-(3). The phase condition being the important one is given in Eq. (5),

$$
w_{o}-w_{s}=\tilde{k} \sqrt{1+\alpha^{2}} \sin \left(w_{s} \tau+\tan ^{-1}(\alpha)\right)
$$

It is apparent from steady-state solution of Eq. (5) that the external feedback rate $\tilde{k}$ modifies threshold carrier density and thus the threshold gain of laser device. If feedback rate is fixed, varying the length of external cavity changes the threshold gain and hence increase or decrease the power output from laser diode. The individual solutions to Eqs. (5) are called as either a mode or an anti-mode of the external cavity. Constructive and destructive interference between the laser diode field and the field reflected from external cavity gives rise to such modes and anti-modes of the system, which are referred to as compound cavity modes of the system [9]. Since the phase condition (5) is a transcendental equation which can only be solved numerically to find $w_{s} \tau$, and the amplitude of $\sin$ term is dictated by the value of $\tilde{k}$, the number of solutions increases or decreases depending the feedback strength increases or decreases. For complete derivations of the rate equations and steady-state solutions, the reader is referred to [1,9-12]. The numerical tool based on above rate equations is developed using Matlab 2016b to study the nonlinear dynamics of single longitudinal mode semiconductor laser subject to optical feedback.

\subsection{Extension of LK Model for Distributed Optical Feedback Regime}

Figure $1 \mathrm{~b}$ shows a laser diode with facet power reflectivities $\mathrm{R}_{1}, \mathrm{R}_{2}$ and the distributed optical feedback section which comprises of $\mathrm{N}$ reflectors with power reflectivitites $\left(\mathrm{R}_{3}, \mathrm{R}_{4}\right.$, $\mathrm{R}_{5}, \ldots, \mathrm{R}_{\mathrm{N}}$ ). The Lang-Kobayashi (LK) model [1] discussed so far has been shown to address external lumped reflector as found in the literature. For the first time, we generalize the LK rate equations [1] to include the impact of distributed feedback (N mirrors) on laser operation and present the corresponding steady state solution,

$$
\begin{gathered}
\frac{d}{d t} \boldsymbol{E}(t)=\frac{1}{2}(1+i \alpha)\left[G_{n}\left(n(t)-\eta_{t h}\right)-\frac{1}{\tau_{p}}\right] \boldsymbol{E}(t)+\sum_{i=1 t o N} \frac{k_{i}}{\tau_{i n}} \boldsymbol{E}\left(t-\tau_{i}\right) e^{-i w_{o} \tau_{i}} \\
w_{o}-w_{s}=\sum_{i=1 \text { toN }} \tilde{k}_{i} \sqrt{1+\alpha^{2}} \sin \left(w_{s} \tau_{i}+\tan ^{-1}(\alpha)\right)
\end{gathered}
$$

where $k_{i}$ and $\tau_{i}$ account for feedback strength and time-delay of 1-to-N reflectors, respectively. The carrier rate equation will remain same as the Eq. (2). The Eqs. (5) and (6) allow to accommodate any arbitrarily number of reflectors provided that the total power reflectivity of all the reflectors remains within the validity range of LK model $(<-30 \mathrm{~dB})$.

The analysis of semiconductor laser subject to reflections from external mirror was peformed using the typical laser and external cavity parameters [12] given in Table 1. 
Table 1 Typical parameters of laser and external cavity used in the analysis of laser subject to optical feedback

\begin{tabular}{lll}
\hline Parameter & Description & Value \\
\hline$\lambda$ & Solitary laser operating wavelength & $1.54 \mu \mathrm{m}$ \\
$l$ & Laser cavity length & $220 \mu \mathrm{m}$ \\
$\eta$ & Refractive index of the lasing mode & 3.8 \\
$R_{1}, R_{2}$ & Power reflectivity of laser facets & 032 \\
$\propto$ & Linewidth-enhancement factor & $\approx 5$ \\
$\varepsilon$ & Nonlinear gain parameter & $3 \times 10^{-3}$ \\
$\xi$ & Differential gain & $2.76 \times 10^{-6} \mathrm{~ns}^{-1}$ \\
$N_{t}$ & Carrier number at the transparency & $1.51 \times 10^{8}$ \\
$\Gamma_{o}$ & Losses in the cavity & $158 \mathrm{~ns}^{-1}$ \\
$\tau_{s}$ & Carrier lifetime & $1 \mathrm{~ns}$ \\
$\eta_{e x t}$ & External cavity refractive index & 1 (air) \\
$L$ & External cavity length & $0-400 \mathrm{~cm}$ \\
$R_{3}$ & External power reflectivity & variable $(-80$ to $-32 \mathrm{~dB})$ \\
\hline
\end{tabular}

\section{Results and Discussion}

\subsection{Lumped Reflector}

Figure 2 shows the behavior of a semiconductor laser diode subject to external optical feedback from a lumped reflector. The steady state solutions of LK model are show in Fig. 2a. We numerically solved phase condition Eq. (5) using typical laser and external reflector cavity parameters given in Table 1 , specifically $\mathrm{L}=8 \mathrm{~cm}$ and $k=0 \mathrm{~dB},-40 \mathrm{~dB}$ and $-34 \mathrm{~dB}$, respectively. As shown in Fig. 2a, the laser device operates at the solitary laser frequency (dotted line) in absence of external feedback $\left(\sqrt{ } R_{3}=0\right)$. With increase in
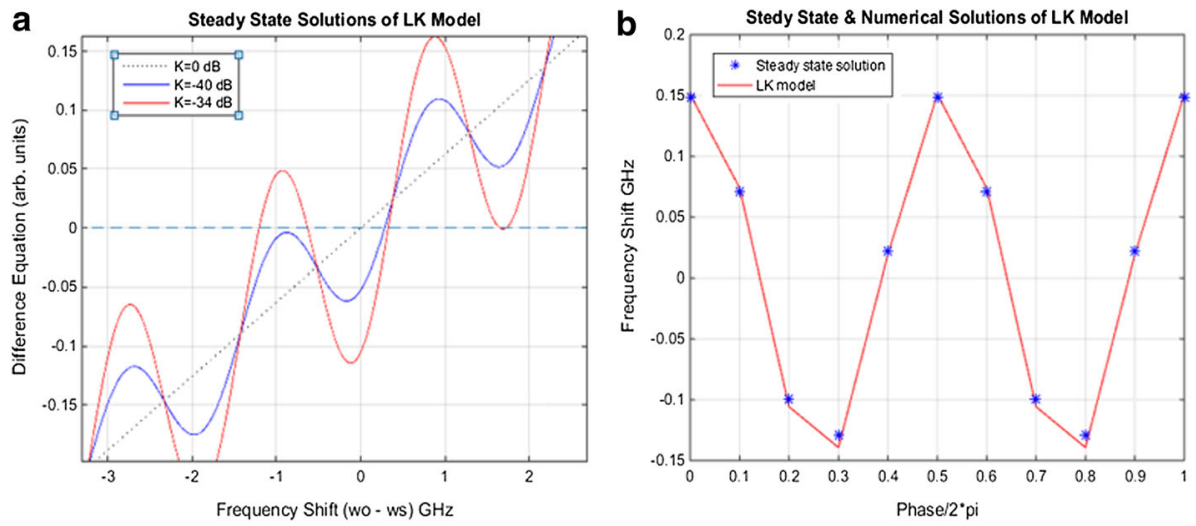

Fig. 2 a Impact of feedback strength $\mathrm{k}$ and $\mathbf{b}$ length of external reflector cavity on the operation of laser diode as predicted by steady-state phase condition (solid red line) and LK model rate equations (blue asterisks). The results agree with each other and prove the reliability of our numerical tool. (Color figure online) 
external feedback strength, the number of solutions increase which reveals the impact of external reflection on the laser device operation. In addition to magnitude of reflection, impact of the phase of the reflected signal (length of the external reflector cavity) on the laser is studied. The output frequency of the laser shifts from the original operating frequency on the order of hundreds of $\mathrm{MHz}$ as displayed in Fig. $2 \mathrm{~b}$, when the phase of the reflection is varied by $2 \pi$, and $k$ and $L$ are taken to be $-60 \mathrm{~dB}\left(\sqrt{ } \mathrm{R}_{3}=0.001\right)$ and $1.54 \mu \mathrm{m}$, respectively. The solutions predicted by two solvers, phase condition Eq. (5) and LK model Eqs. (1-2), agree well as shown in Fig. 2b with solid red line and blue asterisk, and validate our numerical tool developed in Matlab 2016b. Further, it is evident from Fig. 2 that the optical feedback interacts with the field inside the laser cavity and makes it either stable or unstable giving rise to compound cavity modes. The first four regimes [ [1-5], $13,14]$ including laser line-width narrowing or broadening, mode hopping, frequency locking and coherence collapse were observed with change in feedback $k$ and length $\mathrm{L}$ of external cavity which are not discussed here for the sake of brevity.

\subsection{Dynamic Double Cavity System}

In this section, we analyse the dynamic double cavity system. The laser diode is subject to optical feedback from two external reflectors with feedback strengths $\left(k_{1}\right.$ and $\left.k_{2}\right)$ which change position with respect to each other. Here short distance $(\mathrm{L}=1.54 \mu \mathrm{m})$ is assumed between the two reflectors and the laser diode, respectively and change the position of mirrors step by step over $2 \pi$ ( $\lambda$ period). We consider three cases in double cavity system to evaluate the behaviour of the laser. In the first case, feedback power of both the mirrors is equal $\left(k_{1}=k_{2}\right)$ and set to $-41 \mathrm{~dB}$ which makes the maximum feedback $k$ offered to laser device in a double cavity system equal to maximum $-35 \mathrm{~dB}\left(k=k_{1}+k_{2}\right)$, as it depends on the relative phase difference of the two reflectors. Further, in the following cases, we keep the feedback power of two reflectors at different levels, $k_{1} \neq k_{2}$. In the second case, the feedback power reflectivity of reflector 1 is higher $\left(k_{1}=-40 \mathrm{~dB}\right)$ than reflector 2 $\left(k_{2}=-50 \mathrm{~dB}\right)$. In the third case, the feedback power reflectivity of mirror 1 is set less $\left(k_{1}=-50 \mathrm{~dB}\right)$ than mirror $2\left(k_{2}=-40 \mathrm{~dB}\right)$. The relative difference between power reflectivity of the two reflectors is $10 \mathrm{~dB}$. The sum of feedback power offered by two mirrors is $\approx-40 \mathrm{~dB}$.

In Fig. 3a, two-dimensional contour map shows shift in frequency of the laser with phase of reflector 1 and phase of reflector 2 . It is noticed from numerical simulations of double-cavity system with varying phase of the two mirrors $\left(\varnothing_{1}\right.$ and $\left.\varnothing_{2}\right)$ over $2 \pi$ as shown in contour maps that the laser device is stable for all the relative phase difference between the two mirrors and the laser diode. However, the laser locks on to a frequency induced by external cavity configuration and the shift in frequency of laser depends on the relative phase difference.

Figure $3 \mathrm{~b}$, c manifest the case 2 when $k_{1}>k_{2}$ and case 3 when $k_{1}<k_{2}$, respectively. From the contour maps, it is discovered that the emission frequency of the laser is a function of reflector with higher feedback. Nonetheless the laser is still stable for the phase space of the two reflectors $\varnothing_{1}$ and $\varnothing_{2}$. Provided either $k_{1}=k_{2}$ or $k_{1} \neq k_{2}$, following two relations are acquired based on the simulation results from contour maps.

1. When the relative phase difference between 2 external reflectors is $\pi / 2$ or $3 \pi / 2$, the shift in frequency $\Delta f$ is ZERO 

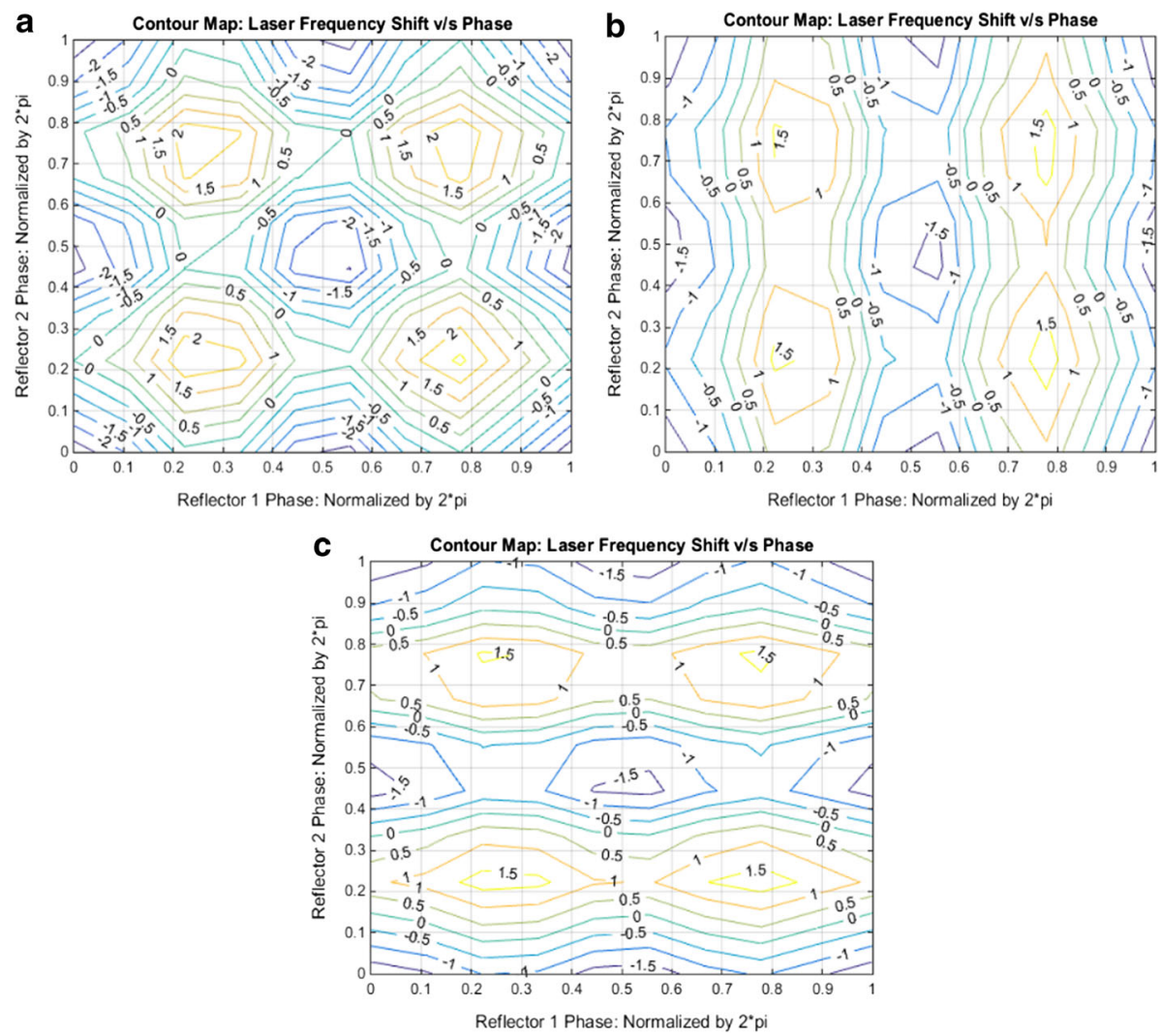

Fig. 3 Contour maps showing the shift in the laser emission frequency versus phase of reflector 1 and reflector 2 when a $k_{1}=k_{2}, \mathbf{b} k_{1}>k_{2}$ and $\mathbf{c} k_{1}<k_{2}$

$$
\left|\emptyset_{1}-\emptyset_{2}\right|= \pm \pi / 2, \quad \pm \frac{3 \pi}{2} \Delta f \rightarrow 0
$$

2. When the relative phase difference between 2 external reflectors is $\pi$ or 0 , shift in frequency $\Delta f$ is $( \pm)$ MAX.

$$
\left|\emptyset_{1}-\emptyset_{2}\right|= \pm \pi, \quad 0 \Delta f \rightarrow \pm \max
$$

We observed the stable output of the laser for the whole phase space considering the length of the two reflectors is up to $1 \mathrm{~cm}$ and for the range of feedback power reflectivity from -80 to $-32 \mathrm{~dB}$ that is within the validity range of LK model. In addition, the shift in frequency $\Delta f$ of the laser with short-scale double cavity is proportional to the sum of the feedback power reflectivity of the two mirrors. Furthermore, it is deduced that when the feedback level of one of the mirror reduces considerably compared to the other, for instance $k_{1} \gg k_{2}$, the frequency shift $\Delta f$ becomes function of the mirror with higher power reflectivity. This work is different from $[9,10]$ in a way that we consider dynamic doublecavity system in which phase of the two reflectors change with respect to each other. 


\subsection{Distributed Optical Feedback Regime}

The term distributed feedback is referred as the spurious optical feedback that originates from a random number of external reflectors as shown in Fig. 1b. In a coherent optical communication system, laser is coupled with fiber optics cable. A similar possible situation is encountered in integrated optical circuits (OICs) in which a laser is integrated into a waveguide on an optical chip. The end of the fiber or the roughness of waveguide in a chip reflects a part of injected light back to the laser. Therefore, the feedback of light can take place from any point on the end of a fiber or portion of a waveguide. All these small points or portion can be perceived as external mirrors which provide multiple time-delays. However, the feedback is continuous (distributed) in nature which means the length of external mirrors is ideally infinite. Therefore, a suitable approach is required to approximate the distributed feedback using finite number of mirrors. In other words, continuous feedback needs to be discretized appropriately, yielding a finite number of reflectors. To achieve a finite number of reflectors, we processed the experimental data of a laser with distributed optical feedback system as follows,

1. To generate even distribution of the experimental data, the data is interpolated, and frequency is translated from given wavelength in the data set,

2. Portions of the broad spectrum are chosen and repeated for periodicity,

3. Fourier transform is computed of the periodic signal to discretize the distributed feedback data,

4. A suitable threshold is set and a finite number of discretized time-samples satisfying the threshold level are stored as external reflectors in a variable space, and the behavior of the laser with distributed optical feedback is examined using our proposed LK model Eqs. $(6,7)$.

A graphical representation of the steps (1 to 4) using the data measured at our Photonics Devices Lab [15] is given in Fig. 4a-e.

Figure $4 \mathrm{a}$ shows the broad spectrum of measured distributed feedback data with the bandwidth of $\approx 50 \mathrm{~nm}$ in frequency domain, which was interpolated in Fig. $4 \mathrm{~b}$ to make the interval between two bins regular, and Fig. 4c shows the overlap before and after the interpolation process. A portion from the broad distributed feedback spectra with the
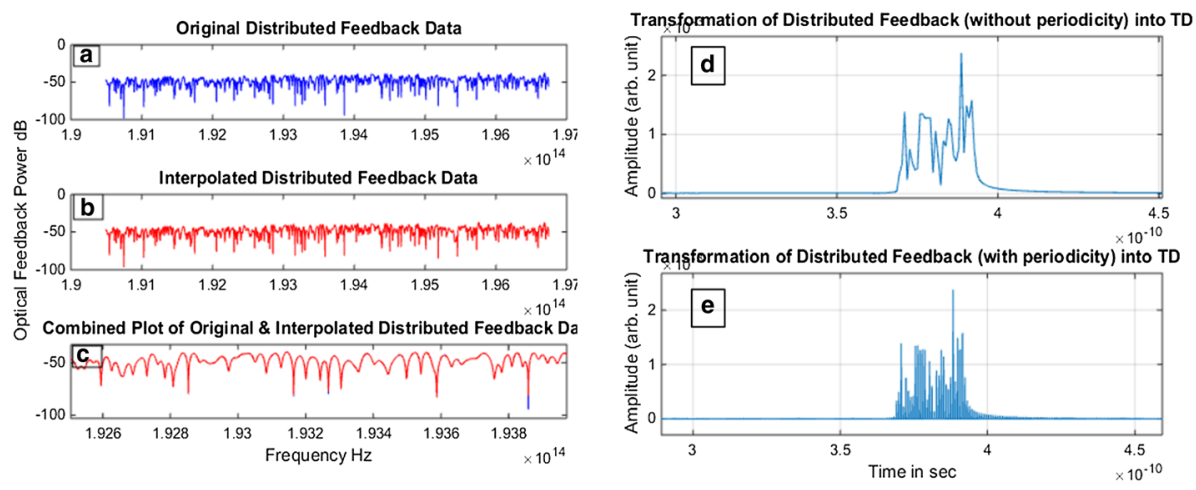

Fig. 4 Processing of measured distributed optical feedback as illustrated in steps 1-4. a Original measured data, b interpolated data, $\mathbf{c}$ before and after interpolation, $\mathbf{d}$ converted data from frequency domain (FD) to time domain using fourier transform (TD) and e TD spectra of periodic waveform to acquire reflectors 
wavelength range of $\approx 5 \mathrm{~nm}$ was selected and repeated for 8 periods. Then, the transformation from frequency domain (FD) to time domain (TD) was materialized. In Fig. 4d, TD transformation without recurrence is calculated, while Fig. 4e is transformation of recurring distributed feedback from FD to TD which gives ideally an infinite impulse response (FIR) like samples. Therefore, we set a threshold to acquire a finite number of FIR samples. In order to determine the quality of approximation, these manipulated FIR samples were converted into FD and the two signals were matched which indicated a good agreement. The absolute threshold value of $2 \times 10^{-5}$ gave 74 FIR samples (distributed reflectors).

Our proposed LK model in Eqs. (6) and (7) allows to accommodate these 74 distributed reflectors provided that the total power reflectivity of all the reflectors remains within the validity range of LK model $(<-30 \mathrm{~dB})$. The maximum feedback generated by 74 distributed single reflections is $-31 \mathrm{~dB}$ (when all reflections are in phase). Figure 5 shows the laser behavior in distributed feedback regime using proposed Eqs. (6) and (7).

It is evident from Fig. 5a that the laser emission frequency is centered on $0 \mathrm{~Hz}$ without feedback, and as the distributed feedback influence is turned on, the frequency of laser shifts by $0.3 \mathrm{GHz}$. It is interesting to note that the laser subject to 74 distributed reflectors operates steadily and locks onto a new frequency. We compared the result of proposed rate Eq. (6) with steady state Eq. (7) of LK model which is shown in Fig. 5b, and remarkably only one solution exists. The frequency shift produced by the two numerical solvers based on proposed LK model are in good agreement with each other.

We took one step further and performed a comparison between distributed feedback regime and lumped reflector by estimating distributed feedback with a single reflector. As shown in Fig. 4e the maximum feedback power in the selected portion of broad distributed feedback spectrum from a reflector is about $-37.5 \mathrm{~dB}$ which is located at time $0.388 \mathrm{~ns}$. In the comparison laser parameters as given in Table 1 were used. A single mirror having feedback power $-37.5 \mathrm{~dB}$ and the phase corresponding to time $0.388 \mathrm{~ns}$ was input in the numerical solvers. The simulation results of the numerical solvers are presented in Fig. 6a, b.

It became clear from the results in Fig. 6a that laser with a single reflector feedback power of $-37.5 \mathrm{~dB}$ is no more stable. This condition of feedback power $(-37.5 \mathrm{~dB})$ is sufficient to induce instability in the laser $[2,13,14]$ and it is confirmed by steady state
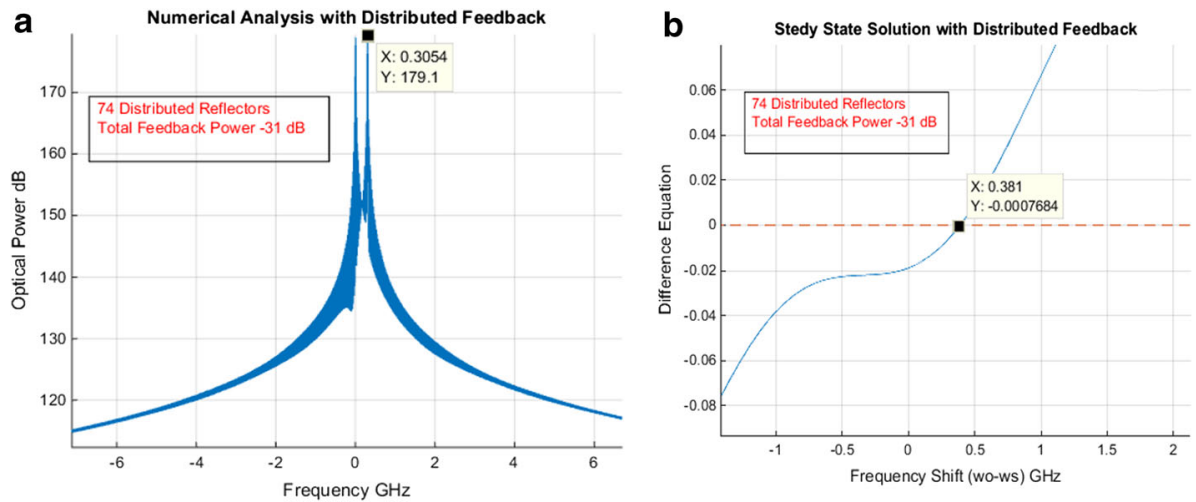

Fig. 5 Numerical analysis of laser in distributed feedback regime (74 reflectors) with a proposed LK rate equations and $\mathbf{b}$ steady-state solver. The results of the two numerical solvers agree with each other 

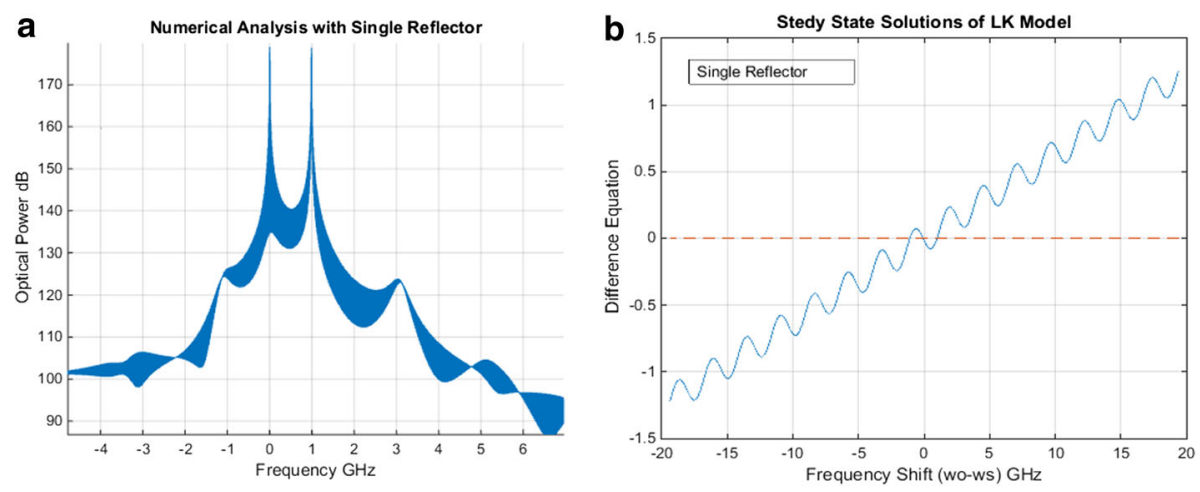

Fig. 6 Numerical analysis of a laser with a lumped reflector having maximum feedback power of $-37.5 \mathrm{~dB}$ solved with a LK model and $\mathbf{b}$ steady-state equation. The laser is unstable compared to distributed feedback regime

solution shown in Fig. 6b. Thus, comparing the distributed optical feedback with single reflector case, we conclude that laser exhibits greater stability when subject to distributed optical feedback. The possible reason of laser stability in distributed optical feedback regime is the effect of phase-cancellation among a large number of reflectors which have impact on the laser emission frequency but do not disturb its stability.

\section{Conclusion}

In conclusion, we have presented a detailed numerical investigation of the effects of optical feedback on the dynamics of a semiconductor laser. Addressing the issue of distributed optical feedback, optical feedback produced by multiple external mirrors has been studied. To this aim we first generalized the LK model to arbitrary number of external reflectors and provided the corresponding steady-state solution, that is done for the first time. Then the dynamic double cavity system was analyzed and conditions for maximum and minimum impact on laser emission frequency have been presented.

It is shown that a laser system in distributed optical feedback regime operates with greater stability and a small shift in emission frequency. However, this conclusion is based on the very parameters used in the numerical analysis. Therefore, this research provides an insight into a new regime of optical feedback which is beneficial for the application of optical integrated devices and opens a new door to further understanding the dynamics of a semiconductor laser device subject to optical feedback. The work can be extended to study the effects of stronger distributed optical feedback $(>-30 \mathrm{~dB})$ using iterative travelling wave model that reduces to LK model in the weak-feedback regime.

\section{References}

1. Lang, R., \& Kobayashi, K. (1980). External optical feedback effects on semiconductor injection lasers properties. IEEE Journal of QE Selected Topics in Quantum Electronics, 16, 347-352.

2. Tkach, R. W., \& Chraplyvy, A. R. (1986). Regimes of feedback effects in $1.5 \mathrm{~nm}$ distributed feedback lasers. Journal of Lightwave Technology, 4(11), 1655-1661.

3. Sano, T. (1994). Antimode dynamics and chaotic intinerancy in the coherence collapse of semiconductor lasers with optical feedback. Physical Review A, 50(3), 2719-2726. 
4. Heil, T., Fischer, I., \& ElsaBer, W. (2001). Dynamics of semiconductor lasers subject to delayed optical feedback: The short cavity regime. Physical Review Letters, 87(24), 243901.

5. Wishon, M. J., Locquet, A., Chang, C. Y., Choi, D., \& Citrin, D. S. (2018). Crisis route to chaos in semiconductor lasers subjected to external optical feedback. Physical Review A, 97(3), 033849.

6. Toomey, J. P., Kane, D. M., McMahon, C., Argyris, A., \& Syvridis, D. (2015). Integrated semiconductor laser with optical feedback: Transition from short to long cavity regime. Optics Express, 23(14), 18754-18762.

7. Zou, Q. (2013). Steady-state behavior of semiconductor laser diodes subject to arbitrary levels of external optical feedback. Optics and Photonics Journal (OPJ), 3(1), 128-134.

8. Panozzo, M., Quintero-Quiroz, C., Tiana-Alsina, J., Torrent, M. C., \& Masoller, C. (2017). Experimental characterization of the transition to coherence collapse in a semiconductor laser with optical feedback. Chaos: An Interdisciplinary Journal of Nonlinear Science, 27(11), 114315.

9. Kane, D., \& Shore, K. A. (2005). Unlocking dyanimcs diversity optical feedback effects on semiconductor lasers. West Sussex: Wiley.

10. Agrawal, G. P. (1984). Line narrowing in a single mode injection laser due to external optical feedback. IEEE Journal of Quantum Electronics, QE-20, 468-471.

11. Ohtsubo, J. (2008). Semiconductor laser stability, instability, and chaos (2nd ed.). Berlin: Springer.

12. Mulet, J., \& Mirasso, C. R. (1999). Numerical statistics of power dropouts based on Lang-Kobayashi model. Physical Review E, 59(5), 5400-5405.

13. Rogister, F., Megret, P., Deparis, O., Blondel, M., \& Erneux, T. (1999). Suppression of low-frequency fluctuations and stabilization of a semiconductor laser subjected to optical feedback from a double cavity: Theoretical results. Optics Letters, 24(17), 1218-1220.

14. Rogister, F., Sukow, D. W., Gavrielides, A., Megret, P., Deparis, O., \& Blondel, M. (2008). Experimental demonstration of suppression of low frequency fluctuations and stabilization of an externalcavity laser diode. Optics Letters, 25(11), 808-810.

15. www.photonics.deib.polimi.it.

Publisher's Note Springer Nature remains neutral with regard to jurisdictional claims in published maps and institutional affiliations.

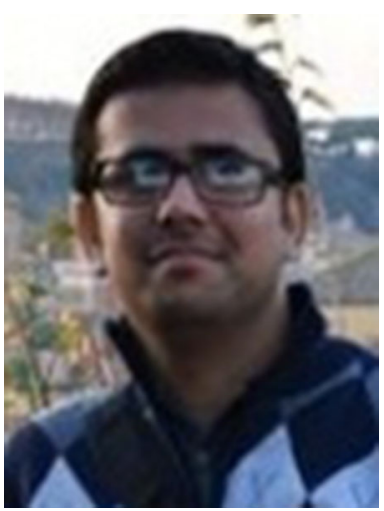

Faisal Ahmed Memon received B.E. in Telecommunication Engineering in May 2011 from Mehran University Pakistan with thesis in optical communications which gave him a drive to explore the world of integrated optics. He was awarded Erasmus Mundus Master's mobility under European Union STRONGTIES project to perform his Master's thesis on Laser Photonics at Politecnico di Milano Italy. Since July 2011, He also serves as Lecturer in Department of Telecommunication Engineering at Mehran University Pakistan. In May 2015, Faisal joined Photonics Devices Group at Politecnico di Milano as $\mathrm{PhD}$ scholar with full scholarship from European Union under Erasmus Mundus LEADERS project and his current research interests are design, fabrication and characterization of micro/nanophotonic devices. 

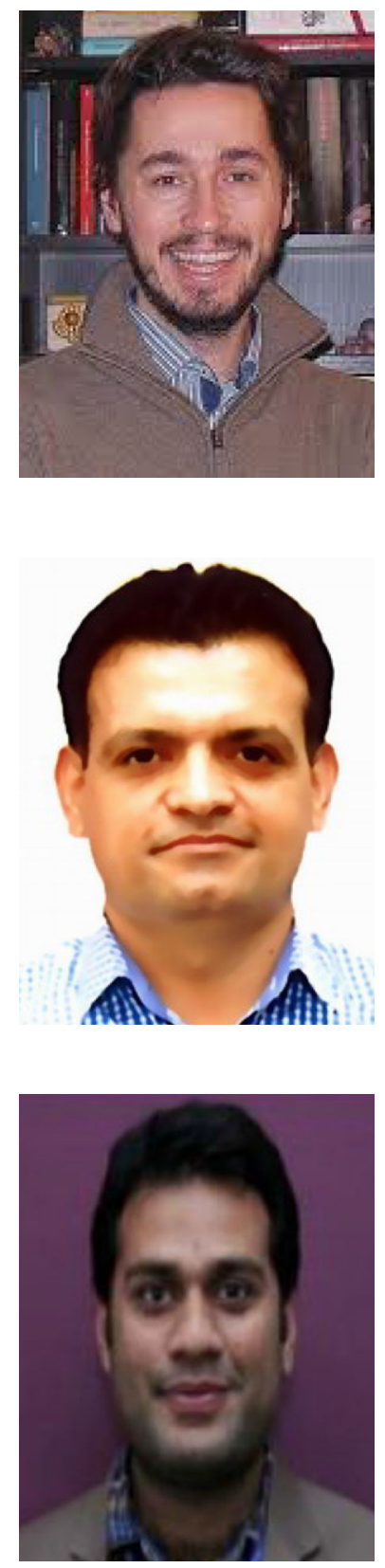

Francesco Morichetti (1976) graduated in 2001 in Telecommunication Engineering (100/100 summa cum laude) at Politecnico di Milano, where he received also the $\mathrm{PhD}$ (summa cum laude) in Information Engineering in 2008. From 2001 to 2008 he was a researcher in the integrated optic staff of Corecom (Consortium for Research in Optical Signal Processing and Switching) in Milan, a research consortium founded in 1996 between Politecnico di Milano and Pirelli SpA. From 2009 to 2011 he worked as a researcher at Fondazione Politecnico di Milano, becoming the Head of the Photonics Devices Lab of Politecnico di Milano. Since 2012 he holds a Post-Doc position at Politecnico di Milano.

Zulfiqar Ali Arain received his M.E degree in communication systems and networks from the Institute of information and communication technologies, Mehran University of Engineering and Technology Jamshoro. Sindh Pakistan. He is currently pursuing his Ph.D. from the Institute of information and communication engineering, Beijing University of Posts and Telecommunication, Beijing China. Presently, he is an Assistant Professor in the Department of Telecommunication Engineering, Mehran University of Engineering and Technology Jamshoro. Pakistan. His research interests include energy-aware networking protocols, wireless networking, multimedia communications, and next-generation Internet Technology.

Umair Ahmed Korai received his masters in telecommunication engineering and management from Mehran University of Engineering and Technology, Jamshoro, Pakistan. He is currently pursuing $\mathrm{PhD}$ from the University of Strathclyde, Glasgow, United Kingdom. He is also working as lecturer at the Mehran University of Engineering and Technology, Jamhsoro, Pakistan (on leave). His research interests include wave propagation, free space optics, fiber optic communication, Photonic integrated circuits. 


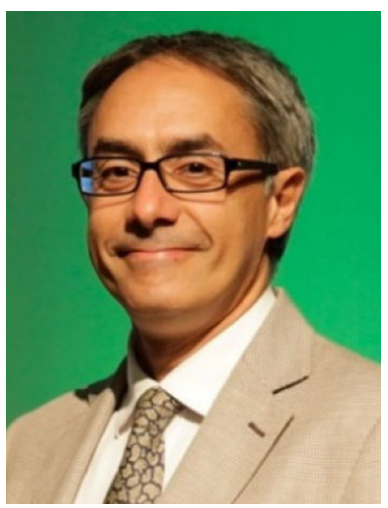

Andrea Melloni is Full Professor at Dipartimento di Elettronica, Informazione e Bioingegneria - Politecnico di Milano - where he leads the group of Photonic Devices and teaches the master course "Photonics Devices". 